\title{
A Plan-view TEM Specimen Preparation Method Using Focused Ion Beam
}

\author{
Lan-Hsuan Lee ${ }^{1}$, Chia-Hao Yu ${ }^{1}$, Yu-Ting Hong ${ }^{1}$ and Cheng-Yen Wen ${ }^{1}$ \\ 1. Department of Materials Science and Engineering, National Taiwan University, Taipei, Taiwan.
}

Focused ion beam (FIB) is a powerful tool for making site-specific and uniform transmission electron microscopy (TEM) specimens out of almost all kinds of solid materials. For thin-film materials, the TEM specimens are usually prepared for cross-sectional and plan-view observations. FIB-based preparation methods for the cross-section specimens have been developed for a long time, and highquality TEM specimens that are suitable for retrieving information with the atomic resolution can be conveniently obtained [1]. On the other hand, reliable plan-view specimen preparation approaches using FIB are seldom reported yet. Using FIB for plan-view specimen preparation is especially suitable for moisture-sensitive materials and two-dimensional atomic layers, such as graphene, $\mathrm{MoS}_{2}$, etc. However, special attentions have to be paid as the thin surface layer can easily be damaged by the ion beam or contaminated by re-deposited material and gallium during preparation [2-6].

In this study, we develop fabrication procedures for preparing plan-view TEM specimens using the FEI Helios 600i DualBeam ${ }^{\mathrm{TM}}$ FIB system, equipped with platinum and carbon gas injection systems and Omniprobe $^{\mathrm{TM}} 200$ manipulating system. The procedures contain three parts: making protecting walls and lid on the sample, lifting out the protected specimen, and final specimen thinning. We apply this method to prepare a plan-view specimen of a single-layer graphene sheet with a graphene adlayer on it. The graphene sheet was grown on a $\mathrm{Cu}$ foil by chemical vapor deposition and transferred to an oxidized Si substrate. The thickness of the oxide layer is $300 \mathrm{~nm}$.

Firstly, the protecting walls and lid are prepared using the material in arbitrary areas on the substrate; they are prepared by making empty wedges surrounding a square area, as shown in Figs. 1(a) and 1(b), respectively, and an additional square hole is milled to make the protecting walls. The lid is lifted out by the manipulator and rotated to an appropriate angle for ion beam milling to shape it into a plate (Fig. 1(c)), and then it is laid onto the protecting walls (Fig. 1(d)). After sealing the gap between the lid and walls, the whole piece is lifted up for ion beam milling to remove the bottom wedge and reveal the walls, as shown in Fig. 1(e). The protecting cap is laid on the graphene sheet (Fig. 1(f)). The cap protects the graphene sheet from ion-beam bombardment and the re-deposition of materials in the following specimen cutting and thinning procedures. The thinning procedures are similar to those in regular FIB cross-section specimen preparation, including lifting out and mounting the specimen onto a $\mathrm{Cu}$ supporting washer (Fig. 1(g)) and ion-beam thinning. When the substrate is thinned down to $\sim 2 \mu \mathrm{m}$, the manipulator is inserted. Its tip is welded with the lid by platinum, and the lid is removed by extracting the manipulator, as shown in Fig. 1(h). The specimen is then further thinned down. Since the ion beam voltage and current $(5 \mathrm{KV}, 15 \mathrm{pA})$ in final thinning procedure is relatively gentle, contamination or ion beam damage on the graphene sheet is not detected.

The SEM image of prepared specimen is shown in Figs. 2(a) and 2(b). In Fig. 2(b), the graphene sheet and the adlayer are still intact after the plan view sample preparation. The existence of the graphene sheet is further confirmed by Raman analysis, and the results indicate that the graphene sheet is monolayer and the adlayer region is a bilayer structure of graphene. The diffraction pattern in Fig. 2(c) shows the reflections due to the hexagonal structure of graphene. In the bilayer area, two sets of the 
hexagonal pattern appear (Fig. 2(d)). The orientation of the graphene adlayer is rotated 20 degrees relative to the underlying graphene sheet.

\section{References:}

[1] J Mayer et al, MRS Bull., 32 (2007), p.400.

[2] R M Langford et al, J. Vac. Sci. Technol., B, 19 (2001), p.755.

[3] H C Floresca et al, Microsc. Microanal., 15 (2009), p.558.

[4] J S Kim et al, Appl. Microsc., 45 (2015), p.195.

[5] K J O'Shea et al, Micron, 66 (2014), p.9.

[6] M Jublot and M Texier, Micron, 56 (2014), p.63.

[7] The authors acknowledge funding from the Ministry of Science and Technology of Taiwan, Grant No. MOST 103-2112-M-002-015-MY3.
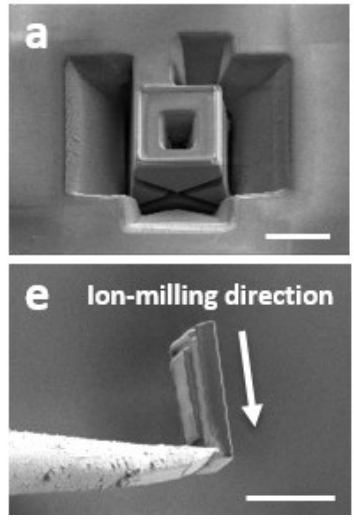
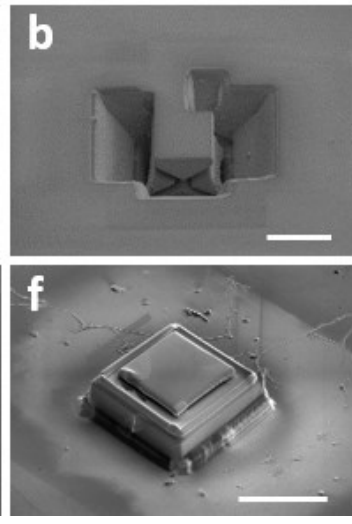
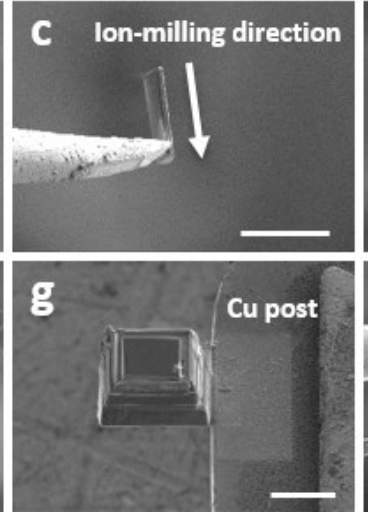
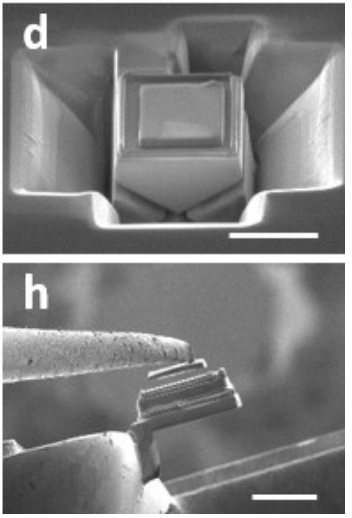

Figure 1. SEM images in the plan-view TEM specimen preparation procedures using focused ion beam (FIB). (a-b) Protecting walls and lid prepared by making empty wedges, respectively. (c) The lid after ion-beam polishing. (d) The walls in (a) covered by the lid in (c). (e) The lifted-out and ion-beam polished protecting cap. (f) The protecting cap on the graphene sheet. (g) The specimen mounted on a $\mathrm{Cu}$ supporting washer. (h) Lifting the lid off the specimen. The scale marker is $10 \mu \mathrm{m}$.
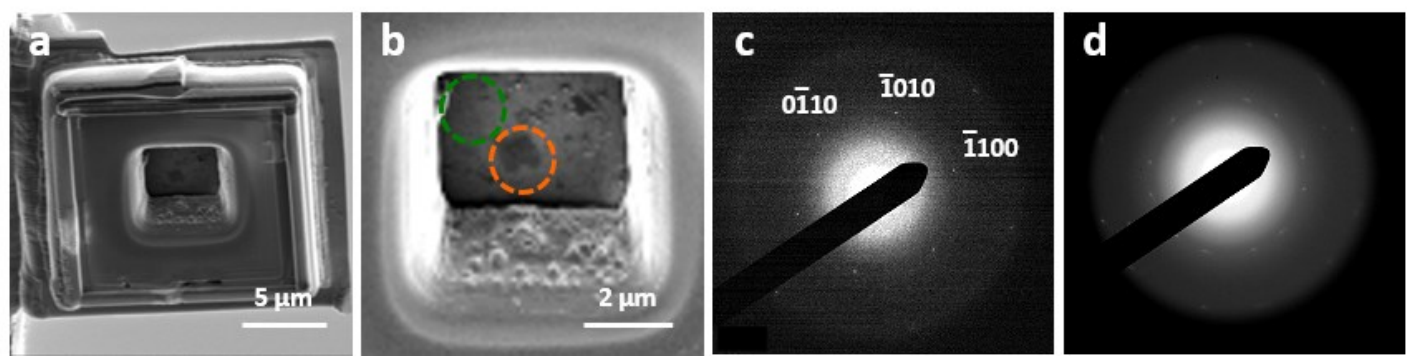

Figure 2. (a-b) SEM images of the plan-view specimen. The graphene sheet is surrounding by the protecting walls. The area label by the green circle is monolayer graphene, and that by the orange circle is bilayer graphene. (c-d) TEM diffraction patterns taken on the green and orange areas, respectively, in (b). 